EPJ Web of Conferences 59, 15005 (2013)

DOI: $10.1051 /$ epjconf/20135915005

(C) Owned by the authors, published by EDP Sciences, 2013

\title{
Laboratory experiments on plasma jets in a magnetic field using high-power lasers
}

\author{
K. Nishio ${ }^{1}$, Y. Sakawa ${ }^{2}$, Y. Kuramitsu ${ }^{2, a}$, T. Morita ${ }^{2}$, T. Ide ${ }^{3}$, M. Kuwada ${ }^{1}$, \\ M. Koga ${ }^{2}$, T. Kato ${ }^{2}$, T. Norimatsu ${ }^{2}$, C. Gregory ${ }^{4}$, N. Woolsey ${ }^{4}$, C. Murphy ${ }^{4}$, \\ G. Gregori ${ }^{5}$, K. Schaar ${ }^{5}$, A. Diziere ${ }^{6}$, M. Koenig ${ }^{6}$, A. Pelka ${ }^{6}$, S. Wang ${ }^{7}$, \\ Q. Dong ${ }^{7}$, Y. $\mathrm{Li}^{7}$ and $\mathrm{H}$. Takabe ${ }^{2}$
}

1 Graduate School of Sci, Osaka Univ, 1-1 Machikaneyama, Toyonaka, Osaka, Japan

2 Institute of Laser Engineering, Osaka Univ, 2-6 Yamadaoka, Suita, Osaka, Japan

3 Graduate School of Engineering, Osaka Univ, 2-1 Yamadaoka, Suita, Osaka, Japan

${ }^{4}$ Department of physics, York Univ, Heslington, York YO10 5DD, UK

${ }^{5}$ Department of physics, Oxford Univ, Clarendon Laborator, Oxford OX1 3PU, UK

${ }^{6}$ LULI Ecole Polytechnique, 91128 Palaiseau Cedex, France

7 Institute of Physics, Chinese Academy of Sciences, Beijing 100190, China

\begin{abstract}
The experiments to simulate astrophysical jet generation are performed using Gekko XII (GXII) HIPER laser system at the Institute of Laser Engineering. In the experiments a fast plasma flow generated by shooting a $\mathrm{CH}$ plane ( $10 \mu \mathrm{m}$ thickness) is observed at the rear side of the plane. By separating the focal spot of the main beams, a non-uniform plasma is generated. The non-uniform plasma flow in an external magnetic field $(0.2 \sim 0.3 \mathrm{~T})$ perpendicular to the plasma is more collimated than that without the external magnetic field. The plasma $\beta$, the ratio between the plasma and magnetic pressure, is $\gg 1$, and the magnetic Reynolds number is $\sim 150$ in the collimated plasma. It is considered that the magnetic field is distorted by the plasma flow and enhances the jet collimation.
\end{abstract}

\section{INTRODUCTION}

Plasma jets are one of the most interesting phenomena in astrophysics. Using X-ray space telescopes (e.g., Einstein, ROSAT, ASCA), high-resolution jet images have been obtained. They have shown detail structures and allowed us to formulate theoretical models. However it is difficult to image time evolution of the astrophysical objects and examine the accuracy of these models because of the long lifetime compared with our time scale. Computer simulations allow the extrapolation from a set of known physical quantities to observations. This approach succeeded in many cases, however, it is not enough to understand many astrophysical problems. Model experiments simulating various astrophysical phenomena have been actively carried out in laboratories. Laboratory simulations of astrophysical phenomena are useful to understand the observations and the simulations. Through the combined efforts of observations, computer simulations, and the laboratory experiments, we can understand astrophysical objects more precisely.

Loupias et al. [1] and Gregory et al. [2] have performed experiments to observe scaling parameters. It is considered that there are three effects to generate astrophysical jets collimation; radiative cooling,

\footnotetext{
${ }^{a}$ e-mail: kuramitsu-y@ile.osaka-u.ac.jp
}

This is an Open Access article distributed under the terms of the Creative Commons Attribution License 2.0, which permits unrestricted use, distribution, and reproduction in any medium, provided the original work is properly cited. 
(a)

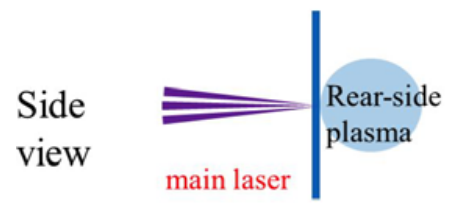

CH Plane target

$3 \mathrm{~mm} \times 3 \mathrm{~mm} \times 10 \mu \mathrm{m}$

Front

view (b)
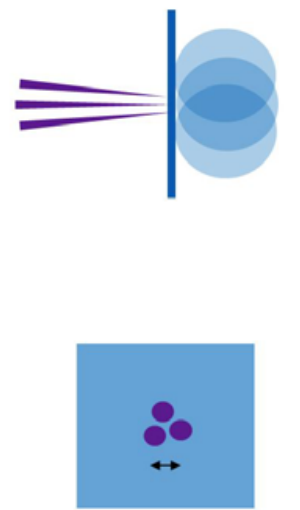

(c)
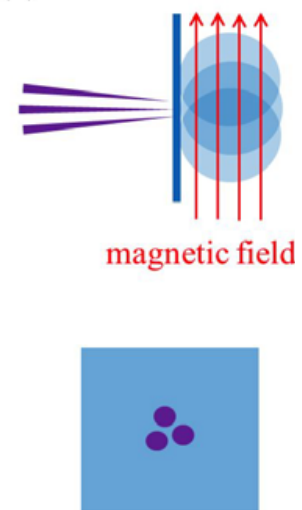

Figure 1. When there is no laser offset, an isotropic plasma is produced. When a laser offset is applied, non-uniform or anisotropic plasmas are produced. Beam separation was $\sim 500 \mu \mathrm{m}$ and focal spot size was $300 \mu \mathrm{m}$. Upper and lower panels show side and front views of experimental conditions, respectively. (a) No offset without external field, (b) with offset without magnetic field, and (c) with offset with an external magnetic field perpendicular to the main laser axis. The $\mathrm{CH}$ plane thickness was $10 \mu \mathrm{m}$. The magnetic field was $0.2 \sim 0.3 \mathrm{~T}$.

interstellar medium, and magnetic field. Shigemori et al. [3] have observed effects of radiative cooling on the collimation of plasma flow. Nicolai et al. [4], Kuramitsu et al. [5], and Gregory et al. [6] have observed effects of interactions between the plasma jet and ambient media. Some experiments to observe interaction between a plasma flow and a magnetic field have been performed by many researchers. Faenov et al. [7] have performed the experiment in a magnetic field $(5 \sim 20 \mathrm{~T})$ parallel to the laser axis. Mostovych et al. [8] have performed the experiment in a magnetic field (1 T) perpendicular to the laser beam axis. In their experiment the plasma flow was created by ablating a solid planar barium target, and the plasma $\beta$ was low $<1$. Plechaty et al. [9] have performed experiments in a magnetic field $(10 \sim 20 \mathrm{~T})$ perpendicular to the laser axis by using high-intensity laser beam. In their experiment the plasma flow was created by ablating a polyethylene plane target, and the plasma $\beta$ was low $<1$. Plasma jets are also observed associated with magnetic reconnections irradiating a solid plane with two beams with beam separation [11-13]. In these experiments the magnetic fields are generated via the laser-plasma interactions, and thus the observed jets are of the scale of the laser, temporally and spatially.

In this paper we investigate experiments in an external magnetic field $(0.2 \sim 0.3 \mathrm{~T})$ perpendicular to the laser axis using a high-power laser system. The plasma flow is created by shooting a $(\mathrm{CH})_{n}$ plane target with multiple beams, and the plasma $\beta$ is $\gg 1$. We focus on the plasma propagation in the presence of the external magnetic field.

\section{EXPERIMENT}

The experiments were performed with GXII laser system at the Institute of Laser Engineering, Osaka university. The laser conditions were as follows: the wavelength was $351 \mathrm{~nm}$, the pulse length was $500 \mathrm{ps}$, the focal spot diameter was $300 \mu \mathrm{m}$, the pulse energy was $\sim 120 \mathrm{~J}$ per beam $\times 3$ beams, the intensity was $(0.2 \sim 3.0) \times 10^{15} \mathrm{~W} / \mathrm{cm}^{2}$.

Figure 1 shows a schematic drawing of the configurations of the main laser beams and the target. We shot $(\mathrm{CH})_{n}$ plane targets (10 $\mu$ m thickness) and observed the fast plasma flow at the rear side of the plane. When there is no beam offset, an isotropic plasma expansion is expected (Fig. 1(a)). To produce nonuniform plasma flow, a $(\mathrm{CH})_{n}$ plane target was irradiated with laser beams with laser offset (Figs. 1(b) 


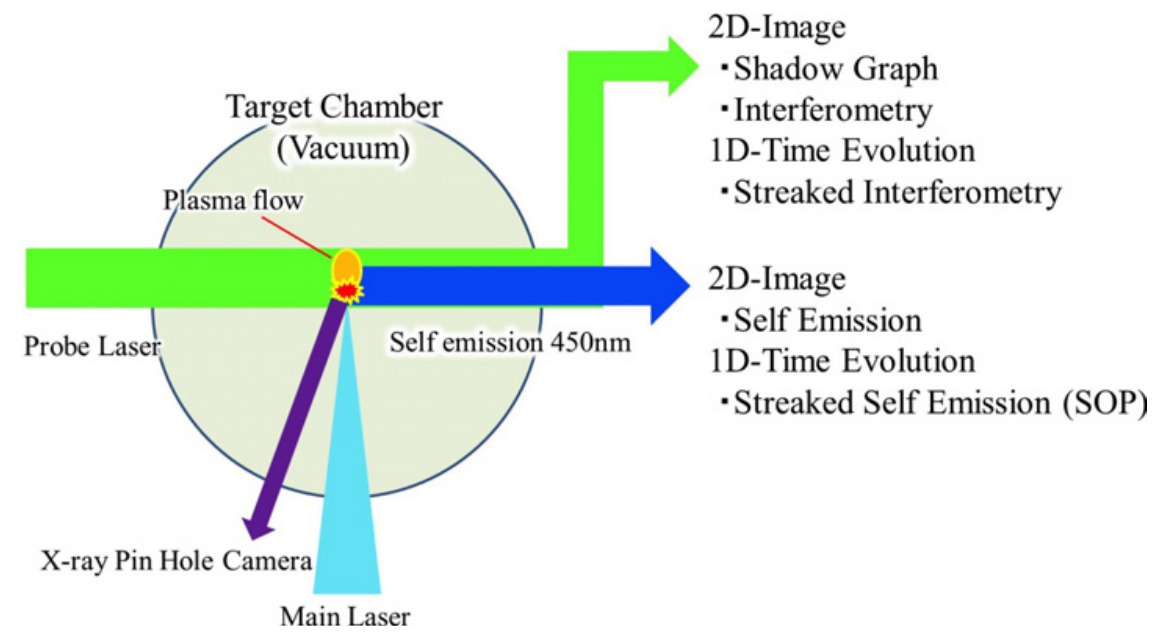

Figure 2. Top view of the target chamber and schematics of the experiment.

and 1(c)). The beam separation was $\sim 500 \mu \mathrm{m}$. To perform the experiments in an external magnetic field, we used a neodymium permanent magnet with $20 \mathrm{~mm}$ in diameter. The external magnetic field was perpendicular to the main laser axis and also perpendicular to plasma flow (Fig. 1(c)). The field strength was $0.5 \sim 0.6 \mathrm{~T}$ at the magnet surface and $0.2 \sim 0.3 \mathrm{~T}$ at the laser axis $(5 \mathrm{~mm}$ from the magnet surface).

The plasmas were observed by the transverse diagnostics with a probe laser and with the selfemission. For the optical probe, the wavelength was $532 \mathrm{~nm}$, and the pulse length was $\sim 14$ ns. Figure 2 shows the target chamber and schematics of diagnostics. With the probe laser we obtained the density information of the two-dimensional snapshots by interferometry and shadowgraphy with ICCD cameras ( $250 \mathrm{ps}$ and $200 \mathrm{ps}$ gate widths, respectively). We also measured the one-dimensional time evolution of the plasma density by streaked interferometry. Using $450 \mathrm{~nm}$ interference filters the self-emission was limited at this wavelength. We obtained the two-dimensional snapshot of the self-emission with ICCD camera (1.6 ns gate width) and time evolution by self-emission streaked optical pyrometer (SOP). We used X-ray pinhole camera to observe focal spot separations.

\section{RESULTS}

Figure 3 shows the interferograms (left), the focal spots images of the main laser beams with X-ray pinhole camera (center), and the density map obtained from the interferograms (right). Figure 3(a) shows the results without offset in the absence of the external field. Without offset all the beams are overlapped and the pinhole image shows a single focal spot. In the interferogram one can see relatively isotropic expansion of the plasma. Figure 3(b) shows the case with beam offset in the absent of the external magnetic field. In the interferogram the plasma flow expands in the direction of the target normal, not isotropically.

Figure 3(c) show the case with beam offset in the presence of the external magnetic field. The plasma is further collimated when compared with Fig. 3(b) in the interferogram. This is more clearly seen in the electron density maps reduced from the interferograms.

The electron density of collimated plasmas was $1.0 \sim 6.0 \times 10^{18} / \mathrm{cm}^{3}$, and flow speed was $\sim 700 \mathrm{~km} / \mathrm{s}$. We estimate the electron temperature is $10 \sim 20 \mathrm{eV}$ and assume ionic charge $\mathrm{Z}$ is 3.5 . The typical plasma size was $5 \sim 10 \mathrm{~mm}$. 
(a)
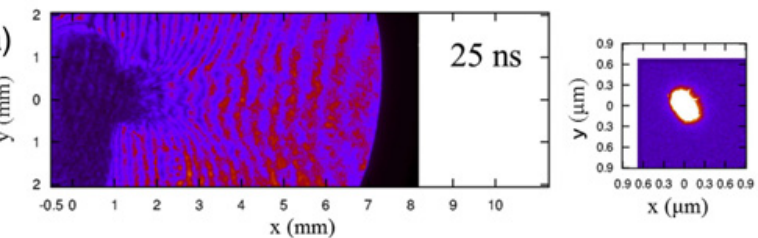

(b)
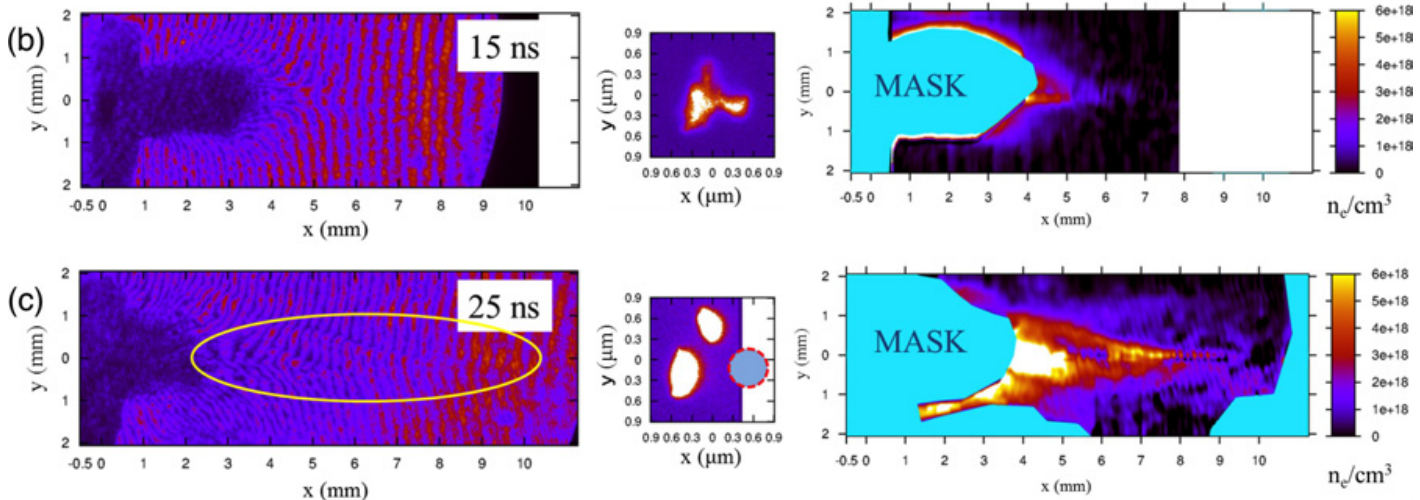

Figure 3. (a) No beam offset without the external magnetic field. (b) With the beam offset without the external magnetic field. (c) With the beam offset with the external magnetic field. The left figures show the interferograms, the center figures show the focal spots images by X-ray pinhole camera, and the right figures show electron density map only for (b) and (c). In the density maps we mask the regions where clear fringes are not seen or the opaque regions at the probe wavelength.

\section{SUMMARY AND DISCUSSIONS}

We investigated jet propagation with and without an external perpendicular magnetic field by irradiating a $\mathrm{CH}$ plane target with high-power laser beams with a laser offset. With the laser offset, the plasma flows in the direction of target normal. With a perpendicular magnetic field, the plasma flow was collimated. Electron Larmor radius was $13 \sim 21 \mu \mathrm{m}$ and ion Lamor radius was $45 \sim 72 \mathrm{~mm}$. It is possible to estimate the magnetic Reynolds number. $\tau_{R}=\mu_{0} L^{2} / \eta$ is resistive diffusion time, $\eta=m_{e} v_{e i \|} / n_{e} e^{2}=5.2 \times$ $10^{-5} Z \ln \Lambda\left(k_{B} T_{e} / e\right)$ is plasma resistivity, $m_{e}$ is electron mass, $v_{e i \|}$ is electron-ion collision frequency, $n_{e}$ is electron density, $e$ is electronic charge, $Z$ is charge state, $\Lambda$ is coulomb logarithm, $k_{B}$ is Boltzmann's constant, $T_{e}$ is electron temperature, $\tau_{H}=L / V$ is hydrodynamic time, $€$ is scale length, $V$ is plasma speed, and $R_{m}=\tau_{R} / \tau_{H}$ is the magnetic Reynolds number. The magnetic Reynolds number was $\sim 150$. Additionally, the plasma pressure was $\sim 5 \times 10^{7} \mathrm{~kg} / \mathrm{s}^{2} \mathrm{~m}$, and the magnetic pressure was $1 \times 10^{4} \mathrm{~kg} / \mathrm{s}^{2} \mathrm{~m}$. The plasma $\beta$, the ratio between the plasma and magnetic pressures was $\gg 1$. In this region of $\beta \gg 1$, the magnetic field is pushed by plasma flow, and in the region of high magnetic Reynolds number, the magnetic field is frozen and considered to move with plasma flow [10]. In our experiment, the plasma flow moved with a magnetic field, and distorted the magnetic field along the plasma flow. The distorted magnetic field is locally parallel to the plasma flow. Thus, along the plasma axis the plasma freely expands along the magnetic field and the distorted field can suppress the lateral expansion of plasma. This will be further discussed elsewhere.

The authors acknowledge the staff at the Institute of Laser Engineering, Osaka University. 


\section{IFSA 2011}

\section{References}

[1] B. Loupias et al., Phys. Rev. Lett. 99, 265001 (2007)

[2] C. D. Gregory et al., Astrophys. J. 676, 420-426 (2008)

[3] K. Shigemori et al., Phys. Rev. E 67, 8838-41 (2000)

[4] Ph. Nicolai et al., Phys. Plasmas 15, 082701 (2008)

[5] Y. Kuramitsu et al., Astrophys. J. Lett. 707, L137-L141 (2009)

[6] C. D. Gregory et al., Phys. Plasmas 17, 052708 (2010)

[7] A. Faenov, V. Dyakin, A. Magunov, T. Pikuz, and Skobelev, Phys. Scripta 53, 591-596 (1996)

[8] A. N. Mostovych, B. H. Ripin and, J. A. Stamper, Phys. Rev. Lett. 62, 24, 2837-2840 (1989)

[9] C. Plechaty et al., H.E.D.P 6, 258-261 (2010)

[10] G. Dimonte and L. G. Wiley, Phys. Rev. Lett. 67, 1755 (1991)

[11] P. M. Nilson et al., Phys. Rev. Lett. 97, 255001 (2006)

[12] P. M. Nilson et al., Phys. Plasmas. 15, 092701 (2008)

[13] J. Y. Zhong, Nat. Phys. 6, 984 (2010) 